\title{
ARTICLE
}

\section{Brain structural correlates of familial risk for mental illness: a meta-analysis of voxel-based morphometry studies in relatives of patients with psychotic or mood disorders}

\author{
Wenjing Zhang $\mathbb{D}^{1}$, John A. Sweeney ${ }^{1,2}$, Li Yao ${ }^{1}$, Siyi $\mathrm{Li}^{1}$, Jiaxin Zeng ${ }^{1}$, Mengyuan $\mathrm{Xu}^{1}$, Maxwell J. Tallman $\mathbb{D}^{2}$, Qiyong Gong $\mathbb{D}^{1}$, \\ Melissa P. DelBello ${ }^{2}$, Su Lui ${ }^{1}$ and Fabiano G. Nery ${ }^{2}$
}

Schizophrenia (SCZ), bipolar disorder (BD), and major depressive disorder (MDD) are heritable psychiatric disorders with partially overlapping genetic liability. Shared and disorder-specific neurobiological abnormalities associated with familial risk for developing mental illnesses are largely unknown. We performed a meta-analysis of structural brain imaging studies in relatives of patients with SCZ, BD, and MDD to identify overlapping and discrete brain structural correlates of familial risk for mental disorders. Search for voxel-based morphometry studies in relatives of patients with SCZ, BD, and MDD in PubMed and Embase identified 33 studies with 2292 relatives and 2052 healthy controls (HC). Seed-based d Mapping software was used to investigate global differences in gray matter volumes between relatives as a group versus HC, and between those of each psychiatric disorder and HC. As a group, relatives exhibited gray matter abnormalities in left supramarginal gyrus, right striatum, right inferior frontal gyrus, left thalamus, bilateral insula, right cerebellum, and right superior frontal gyrus, compared with HC. Decreased right cerebellar gray matter was the only abnormality common to relatives of all three conditions. Subgroup analyses showed disorder-specific gray matter abnormalities in left thalamus and bilateral insula associated with risk for SCZ, in left supramarginal gyrus and right frontal regions with risk for $\mathrm{BD}$, and in right striatum with risk for $\mathrm{MDD}$. While decreased gray matter in right cerebellum might be a common brain structural abnormality associated with shared risk for SCZ, BD, and MDD, regional gray matter abnormalities in neocortex, thalamus, and striatum appear to be disorder-specific.

Neuropsychopharmacology (2020) 45:1369-1379; https://doi.org/10.1038/s41386-020-0687-y

\section{INTRODUCTION}

Major psychiatric disorders, such as schizophrenia (SCZ), bipolar disorder (BD), and major depressive disorder (MDD), are highly heritable [1-3]. The estimated heritability of SCZ, BD, and MDD is $81 \%, 85 \%$, and $37 \%$, respectively [1-4]. It is well established that first-degree relatives of patients with these psychiatric disorders have an increased risk for developing the same condition $[2,3,5,6]$. The increased risk for developing a psychiatric disorder among relatives extends beyond the disorder present in the proband to other disorders as well [7-9]. Moreover, the genetic relationship estimated from genome-wide single-nucleotide polymorphisms was found to be high between SCZ and BD, and moderate between BD and MDD [10]. These findings suggest that psychiatric disorders are not discrete biological entities but rather share common neurobiological pathways. In fact, increasing interests exist in identifying transdiagnostic biosignatures of psychopathology that may help to increase diagnostic accuracy and preventative strategies [11-14].

Identifying neuroimaging biomarkers of common signatures for neuropsychiatric disorders, as well as for the genetic risk, gains growing attention. Brain morphometric deficits of gray matter, which are more like trait-related features with high stability $[15,16]$, may be particularly preferred in providing quantitative measures of the brain in vivo. Recent efforts have investigated common neuroanatomical structural abnormalities in patients with SCZ, BD, and MDD. For instance, a meta-analysis of 193 studies comprising individuals with SCZ, BD, MDD, substance use disorders, obsessive-compulsive disorder (OCD), and anxiety disorders found that decreased gray matter in the dorsal anterior cingulate and insula were common across the disorders [17]. Another study comparing patients across SCZ, BD, and MDD found these three psychiatric conditions shared gray matter loss in paralimbic and heteromodal regions, including the orbitofrontal cortex, insula, parahippocampal gyrus, and cingulate gyrus [18]. A recent study in unmedicated patients found that patients with first-episode psychosis, MDD, posttraumatic stress disorder, and OCD showed increased gray matter volume (GMV) in the bilateral putamen [19]. These findings of gray matter changes in the same brain regions across major psychiatric disorders support the hypothesis that there are biological abnormalities that are shared across psychiatric disorders and others that differentiate them.

Studies of relatives of patients have shown a similar pattern, though with less severe manifestations than in patients with $\mathrm{SCZ}$, $\mathrm{BD}$, or MDD [20-23]. Meta-analysis of structural imaging studies have reported that relatives of patients with BD show increased GMV in the right inferior frontal gyrus and left superior temporal

\footnotetext{
${ }^{1}$ Huaxi MR Research Center (HMRRC), Department of Radiology, Functional and molecular imaging Key Laboratory of Sichuan Province, West China Hospital, Sichuan University, Chengdu 610041, China and '2Department of Psychiatry and Behavioral Neuroscience, University of Cincinnati College of Medicine, Cincinnati, OH 45219, USA

Correspondence: Su Lui (lusuwcums@tom.com) or Fabiano G. Nery (neryfo@ucmail.uc.edu)
}

Received: 18 January 2020 Revised: 19 April 2020 Accepted: 22 April 2020

Published online: 30 April 2020 


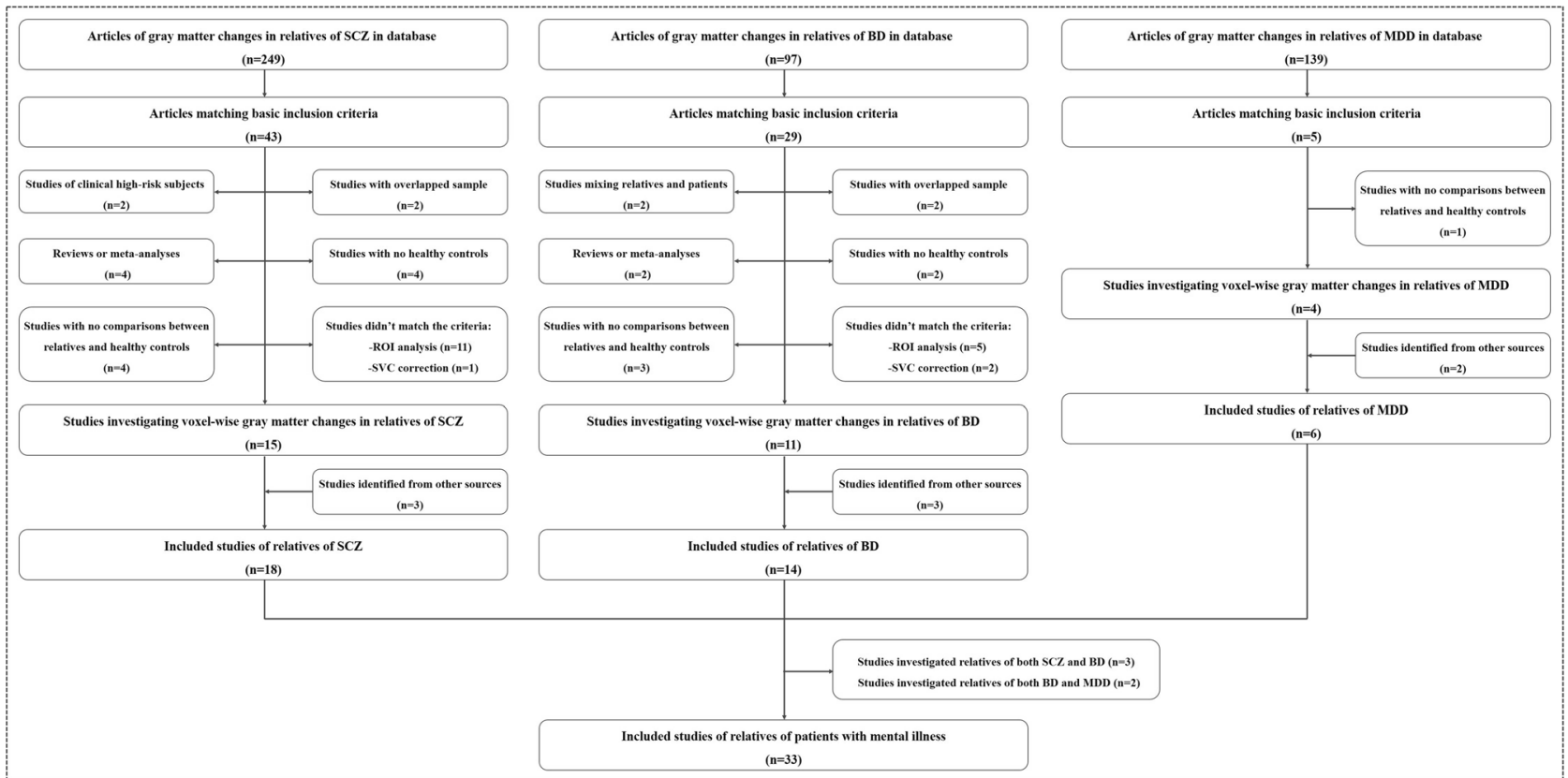

Fig. 1 The flowchart of the selecting procedures of the current meta-analysis. BD bipolar disorder, MDD major depressive disorder, SCZ schizophrenia.

gyrus, and reduced GMV in the right lingual gyrus compared with healthy controls (HC) [21]. In individuals at high risk for psychosis, decreased gray matter was found in the left parahippocampal gyrus, bilateral anterior cingulate cortex, right superior temporal gyrus, left insula, and left cerebellum compared with $\mathrm{HC}$ [23]. In a recent multisite meta-analytic study, compared with HC, first-degree relatives of patients with SCZ had smaller thalamic volumes, but those of patients with BD had larger intracranial volumes, supporting a notion for distinct biosignatures of risk for each condition besides the overlaps in genetic liability [22]. However, this study used global and subcortical measures extracted at each participating site of predefined regions of interest (ROI), did not include relatives of patients with MDD, and did not examine differences across disorders. Therefore, the differentiation of distinct and overlapping gray matter abnormalities across disorders in relatives of patients with $\mathrm{SCZ}, \mathrm{BD}$, or MDD remains uncertain.

In order to address this issue, we thus conducted a metaanalysis of voxel-based structural gray matter studies in relatives of patients with SCZ, BD, and MDD. Our primary goal was to identify common gray matter abnormalities that are shared across relatives of the three major psychiatric disorders. To accomplish this goal, we first compared relatives of all three psychiatric disorders against $\mathrm{HC}$, and subsequently relatives of each psychiatric disorder against HC.

\section{METHODS AND MATERIALS}

\section{Selection procedures}

Two experienced researchers independently searched PubMed (http://www.ncbi.nlm.nih.gov/pubmed) and Embase (https://www. embase.com) to identify all eligible imaging studies in relatives of patients with $\mathrm{SCZ}, \mathrm{BD}$, or MDD published in English before February 2020. We used the following keywords to identify potentially appropriate studies: (1) BD, SCZ, depression, or depressive disorder or MDD; (2) family, families, relative, sibling, parent, offspring, and twin; and (3) GMV or gray matter density (GMD). The reference lists of the selected original articles or meta-analyses of $\mathrm{BD}, \mathrm{SCZ}$, and MDD were manually checked for additional qualified studies.

We included all studies that met the following criteria: (1) original papers published in peer-reviewed journals; (2) conducted anatomic GMV/GMD analysis at the whole brain level; (3) included comparisons between relatives of patients with $\mathrm{SCZ}, \mathrm{BD}$, or $\mathrm{MDD}$, and $\mathrm{HC}$; and (4) localized effects using MNI or Talairach coordinates. Studies were excluded if (1) they were reviews or meta-analyses; (2) they exclusively used an ROI approach, partial brain coverage, or small volume correction; (3) peak coordinates of effects could not be retrieved; (4) they had overlapping reports of data (e.g., overlapping samples from the same institution or authors reported in different papers, in which case the study with the largest sample was included). Corresponding authors were contacted for details that were required for the present metaanalysis, if necessary. We followed the Meta-analysis Of Observational Studies in Epidemiology guidelines [24] for our present study. The flowchart of searching procedures is showed in Fig. 1.

In some studies, more than one dataset of relatives of patients with the same disorder existed. Nery et al. divided the offspring of $\mathrm{BD}$ into symptomatic and asymptomatic ones [25]. Another study on relatives of $\mathrm{BD}$ patients was a two-center study with a replication design, which also separated affected and unaffected relatives, and performed four different comparisons [26]. There were other studies that separated SCZ relatives into familial and sporadic ones [27], or into those from the same families as probands that were also studied and from other families [28], or into relatives of deficit and nondeficit SCZ [29]. For these studies, two or more datasets were obtained and treated as separate samples.

Quality assessment of selected studies

The quality of each selected study was evaluated with a 12-point checklist as used in previous neuroimaging meta-analyses [30, 31]. This checklist was modified to assess critical variables that were important to our meta-analysis (see Supplementary Materials), which is suitable especially for neuroimaging studies. It is noteworthy that this checklist was adopted just to rate the completeness of the reported methods and results, without criticizing the work itself or investigators.

Meta-analysis of gray matter abnormalities in relatives of psychiatric patients

The Seed-based d Mapping (SDM, formerly Signed Differential Mapping, www.sdmproject.com) was used as the primary toolbox 
for our meta-analyses. This software has been widely used and successfully validated in previous studies [32-36]. Its advantageous features have also been reported elsewhere [34-36] and are further presented in the Supplementary Materials. The procedures of data analysis within SDM include four main steps: data preparation, preprocessing, mean analysis, and family-wise error rate (FWE) correction. Details are also showed in the Supplementary Materials.

The age span was wide in studies of offspring, siblings, and parents of individuals with the target psychiatric disorders (ranging from 11.0 to 54.8 years old on average). Thus, we controlled for age in the main meta-analysis and in all subsequent subgroup analyses.

Post hoc replication analysis

Given that some studies had a sample size $\leq 10$ for target population [27, 37, 38], first post hoc analysis was conducted within studies that enrolled over ten relatives of patients with a psychiatric disorder (31 studies with 40 samples).

Most of the studies (27 studies with 30 samples) included relatives of psychiatric patients who were healthy or unaffected at the time of study participation (unaffected relatives, see Table S1), while few (four studies with five samples) included relatives who exhibited psychiatric diagnoses (affected relatives, see Table S2). To remove effects of psychiatric diagnoses in relatives, we also conducted a replication analysis with only the unaffected relative groups. Moreover, not all the studies included in our meta-analysis adopted a multiple comparison correction step such as false discovery rate or FWE, we thus conducted a second replication analysis with only the studies that controlled for multiple comparisons (25 studies with 31 samples).

Disorder-specific subgroup meta-analysis of relatives

To further investigate the specificity of our findings with regards to each psychiatric disorder as opposed to their transdiagnostic nature, we performed secondary analyses to characterize gray matter abnormalities within relatives of $S C Z, B D$, and MDD separately. Of note, one study included relatives of both MDD and BD in the same group [39], thus was not included in the subgroup analyses.

\section{Analysis of heterogeneity and publication bias}

We tested the between-studies heterogeneity of individual clusters identified in the primary meta-analyses using a randomeffects model with $Q$ statistics. Magnitude of heterogeneity was estimated using $I^{2}$ index, computed as $100 \% \times(Q-d f) / Q$, where $\mathrm{df}$ is the degree of freedom that estimates the proportion of variability due to nonrandom differences between studies. We also created funnel plots of the peaks of the main findings and examined the possibility of publication bias for altered regions using the Egger's test in SDM. Statistical significance was determined with FWE corrected $p$ values $<0.05$.

Meta-regression analysis

To identify potential effects of demographic and methodological variables on gray matter abnormalities in relatives, meta-regression analyses were conducted between the gray matter abnormalities found in the primary meta-analyses and sex (male \%), MRI field strength, acquisition slice thickness, and smoothing kernel of full width at half maximum (FWHM) with general linear regression model. The regression map was corrected using FWE.

\section{Additional analyses}

With an exploratory purpose, we have also conducted the analysis with the relatives of patients with $\mathrm{SCZ}$ and $\mathrm{BD}$ as a whole given the heritability variance of MDD is about one-third compared with that of SCZ and BD. We also explored the dimensional aspects of familial risk for BD and MDD combined as mood disorders. Details are in Supplementary Materials.

\section{RESULTS}

Included studies

We included 33 structural MRI studies, with 44 comparisons and related datasets, that comprised 2292 relatives (mean age: $30.25 \pm$ 10.58 years, male/female $=1016 / 1276$ ) of patients with a psychiatric disorder, and $2052 \mathrm{HC}$ (mean age: $29.29 \pm 8.86$ years, male/ female $=970 / 1082$ ) in our meta-analysis. Among these studies, two enrolled relatives of patients with $\operatorname{BD}$ and $\operatorname{MDD}[38,39]$, while another three studied relatives of $\mathrm{BD}$ and $\mathrm{SCZ}$ patients simultaneously [40-42]. As a result, there were respectively 18, 14, and 6 studies that investigated gray matter abnormalities in relatives of patients with SCZ $(n=1389$, mean age: $32.50 \pm 9.46), \mathrm{BD}(n=$ 719 , mean age: $26.38 \pm 11.60)$, and $\operatorname{MDD}(n=184$, mean age: $28.38 \pm 9.66)$. The mean quality scores of included studies for SCZ, BD, and MDD were 11.0 (range 9.5-12), 11.4 (range 10.5-12), and 11.5 (range 11-12) separately, showing that the included studies were of high and similar quality across disorders.

To keep our scope focused on identifying effects of familial risk on gray matter rather than effects of established illness, and due to the fact that 11 studies (33.3\%) did not include proband's imaging data, we report here our primary focus, which is comparisons between relatives versus controls, whereas additional exploratory comparisons between patients versus relatives and patients versus $\mathrm{HC}$ are showed in Supplementary Materials. Demographic characteristics of study participants, as well as methodological details and quality scores of included studies, are summarized in Table 1.

Gray matter abnormalities in overall relatives versus HC

Our main analyses showed that, as a group, relatives of patients with SCZ, BD, and MDD exhibited increased gray matter in the left supramarginal gyrus, right striatum, right inferior frontal gyrus, and left thalamus, and decreased gray matter in the left insula extending to temporal regions, right cerebellum, right superior frontal gyrus, and right insula compared with HC (Table 2, Fig. 2). Most of these findings were replicated in the post hoc analysis within studies that had a sample size of relatives over ten (2253 relatives versus $1998 \mathrm{HC}$ ), except that the gray matter decrease of right insula was missing (Table S3). In post hoc replication analyses with unaffected relatives (1320 relatives versus $1710 \mathrm{HC}$ ), the same alterations were observed as in the primary analysis (Table S4).

The post hoc analysis within studies that used additional correction steps (1822 relatives versus $1744 \mathrm{HC}$ ) confirmed the findings from the main analyses, with the exception of increased gray matter in right striatum and left thalamus in relatives versus HC (Table S5). They were no longer significant, suggesting that those abnormalities might be less robust, given that those were lost when including only studies with correction for multiple comparisons.

Disorder-specific gray matter abnormalities in relatives versus $\mathrm{HC}$ In secondary analyses in relatives of patients with each psychiatric diagnosis considered separately, we found that relatives of patients with SCZ (1389 relatives versus $1487 \mathrm{HC}$ ) showed increased gray matter in the left thalamus, and decreased gray matter in the left middle temporal gyrus, right cerebellum, and right insula compared with HC (Fig. 3, Table S6). Relatives of patients with BD (709 relatives versus $729 \mathrm{HC}$ ) showed increased gray matter in the right inferior frontal gyrus, left supramarginal gyrus, right gyrus rectus, and decreased gray matter in the right cerebellum and the right superior frontal gyrus compared with $\mathrm{HC}$ (Fig. 3, Table S7). The relatives of patients with MDD (157 relatives versus $210 \mathrm{HC}$ ) showed increased gray matter in the left 


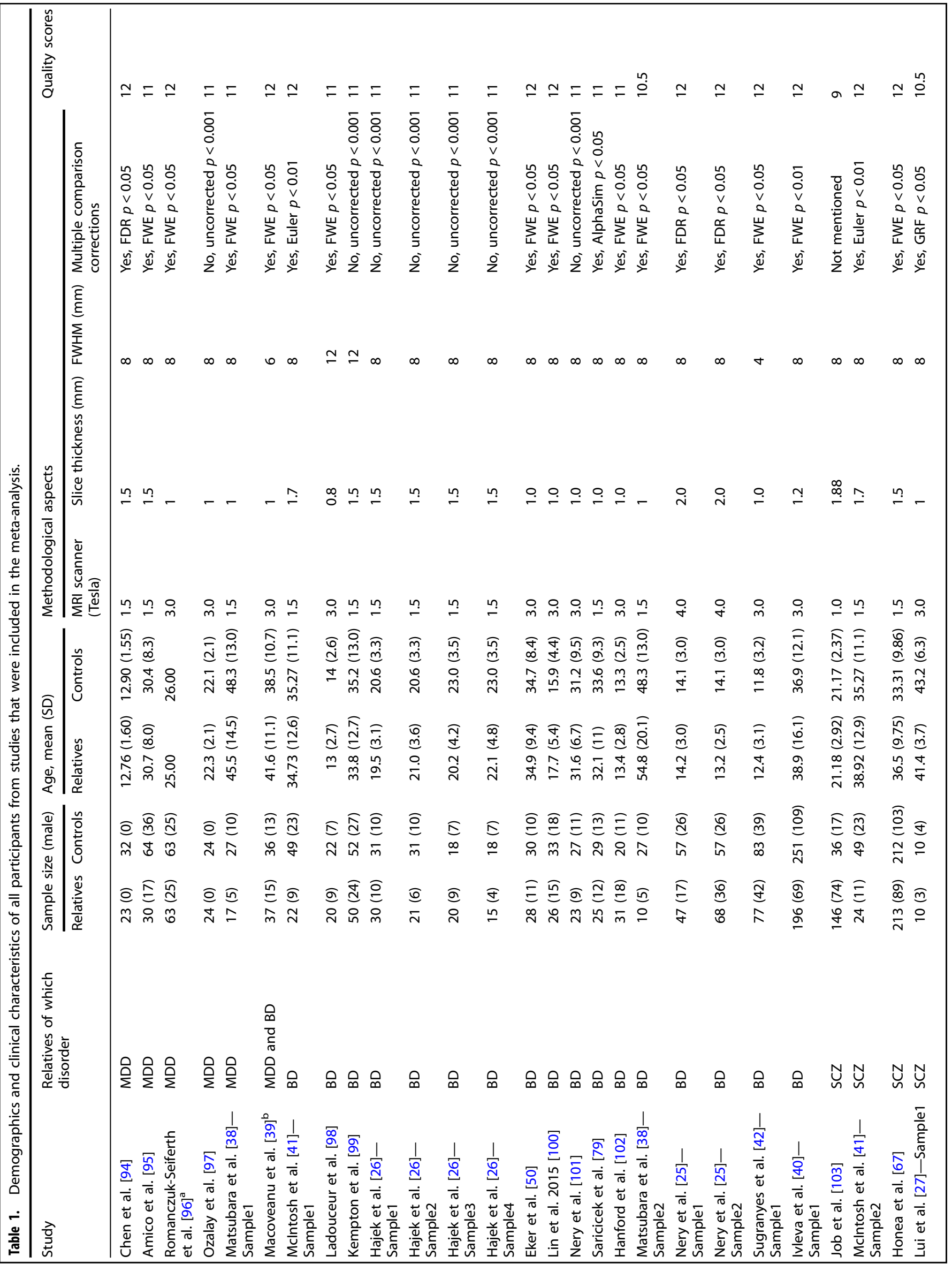




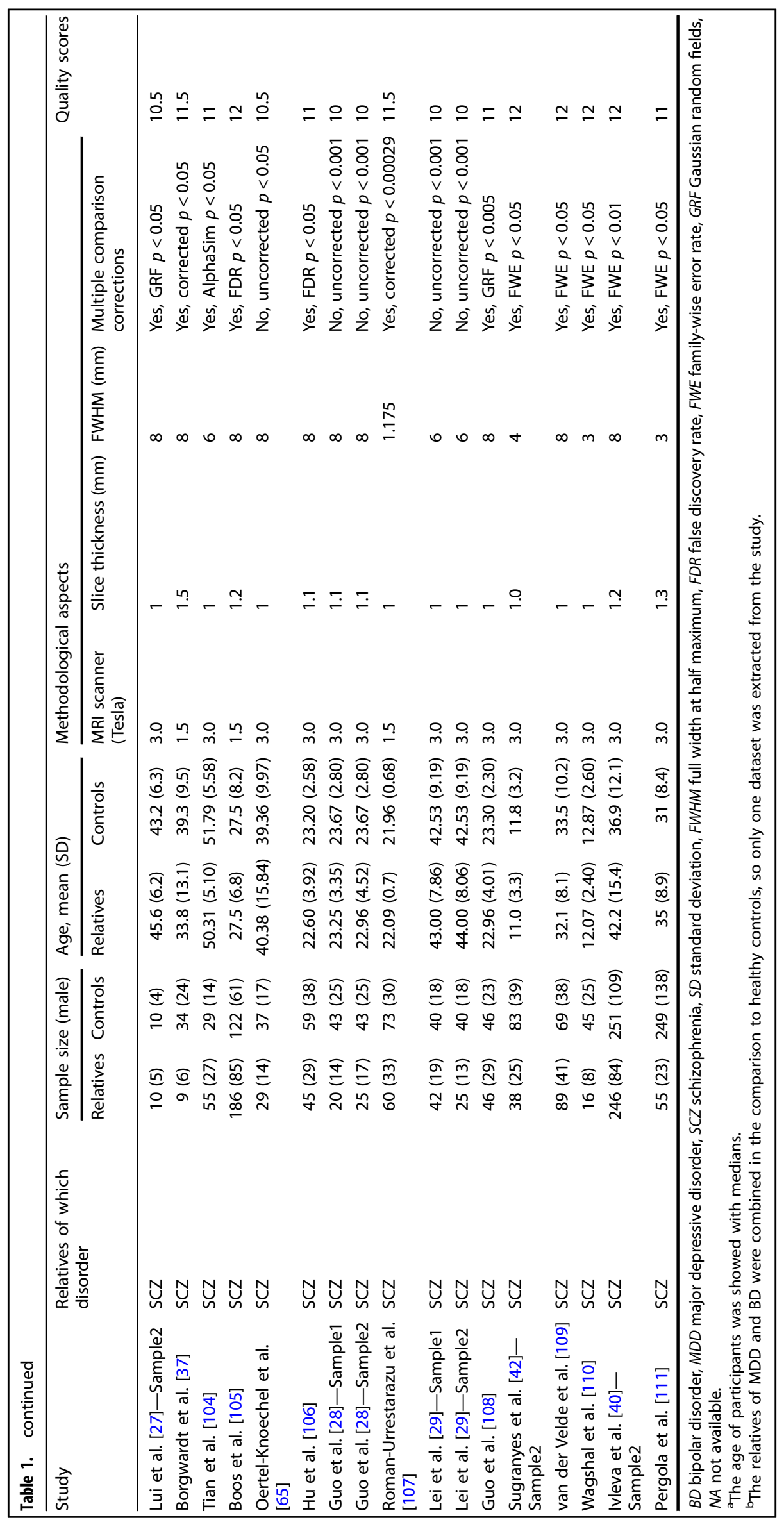


Table 2. Gray matter abnormalities in overall relatives for all the three psychiatric disorders.

\begin{tabular}{|c|c|c|c|c|c|}
\hline Region & $\mathrm{MNI}, x, y, z$ & SDM-Z & $p$ value & Voxels & Cluster breakdown (no. of voxels) ${ }^{a}$ \\
\hline L supramarginal gyrus & $-54,-48,26$ & 3.88 & $<0.001$ & 722 & $\begin{array}{l}\text { L supramarginal gyrus }(269) ; \\
\text { L superior temporal gyrus ( } 219) ; \\
\text { L angular gyrus ( } 87 \text {; } \\
\text { L middle temporal gyrus (65) }\end{array}$ \\
\hline $\mathrm{R}$ striatum & $26,14,-2$ & 3.50 & $<0.001$ & 378 & $\begin{array}{l}\text { R striatum (177); } \\
\text { R gyrus rectus ( } 42) \text {; } \\
\text { R superior frontal gyrus, orbital part (36); } \\
\text { R inferior frontal gyrus, orbital part (21) }\end{array}$ \\
\hline $\mathrm{R}$ inferior frontal gyrus & $46,24,-6$ & 2.26 & 0.012 & 219 & $\begin{array}{l}\mathrm{R} \text { inferior frontal gyrus, orbital part (79); } \\
\mathrm{R} \text { inferior frontal gyrus, triangular part (39); } \\
\mathrm{R} \text { insula (11) }\end{array}$ \\
\hline$L$ thalamus & $-10,-16,4$ & 2.62 & 0.004 & 79 & $L$ thalamus $(79)$ \\
\hline \multicolumn{6}{|l|}{ Relatives $<$ healthy controls } \\
\hline \multirow[t]{2}{*}{$\mathrm{R}$ cerebellum } & $10,-52,-12$ & -5.92 & $<0.001$ & 1593 & $\begin{array}{l}\text { R lingual gyrus (349); } \\
\text { Cerebellum, vermis lobule IV/V (303); } \\
\text { R cerebellum, hemispheric lobule IV/V (297); } \\
\text { Cerebellum, vermis lobule VI (178); } \\
\text { R cerebellum, hemispheric lobule VI (87); } \\
\text { R fusiform gyrus (79); } \\
\text { R cerebellum, hemispheric lobule III (15); } \\
\text { L cerebellum, hemispheric lobule IV/V (42); } \\
\text { L cerebellum, hemispheric lobule VI (29) }\end{array}$ \\
\hline & $24,-32,-28$ & -2.84 & 0.002 & 108 & $\begin{array}{l}\text { R cerebellum, hemispheric lobule IV/N (42); } \\
\text { R cerebellum, hemispheric lobule III (12) }\end{array}$ \\
\hline
\end{tabular}

$L$ left, $R$ right.

anly regions with cluster size larger than 10 voxels are reported.

parahippocampal gyrus and right striatum, and decreased gray matter in the right cerebellum compared with HC (Fig. 3, Table S8).

Heterogeneity and publication bias

Among regions with gray matter abnormalities, right striatum, right inferior frontal gyrus, left thalamus, and right insula showed significant between-study heterogeneity (all with FWE corrected $p<0.05$, Table S9). The Egger's test of funnel plot asymmetry did not identify any evidence of publication bias in any cluster $(p>0.05)$.

\section{Meta-regression findings}

The whole brain meta-regression analysis showed that studies with fewer male patients showed more gray matter abnormalities in the right inferior frontal gyrus (peak $\mathrm{MNI}=48,28,-4, z=-2.27, p=$ $0.017,203$ voxels). As for methodological variables, studies using lower field strength MRI scanners reported greater gray matter abnormalities in the right inferior frontal gyrus (peak $\mathrm{MNI}=40,32$, $-10, z=-3.45, p<0.001,1050$ voxels), while larger slice thickness was associated with higher increases of gray matter in right inferior frontal gyrus (peak $\mathrm{MNI}=42,32,-10, z=2.46, p=0.002,919$ voxels) and right superior frontal gyrus (peak $\mathrm{MNI}=4,52,-10, z=$ 2.29, $p=0.032,28$ voxels). The findings above survived FWE correction with threshold-free cluster enhancement. No significant associations were found between any other gray matter abnormality and sex, field strength, smoothing FWHM, or slice thickness.

Additional analysis and findings

The findings of analysis with relatives of patients with SCZ and BD as a whole are showed in Table S10, while findings for relatives of patients with mood disorders including BD and MDD are showed in Table S11. The details and findings of comparisons between patients and relatives, and between patients and $\mathrm{HC}$ are showed in Table S12-15.

\section{DISCUSSION}

To the best of our knowledge, this is the first voxel-based whole brain structural imaging meta-analysis that attempted to identify 


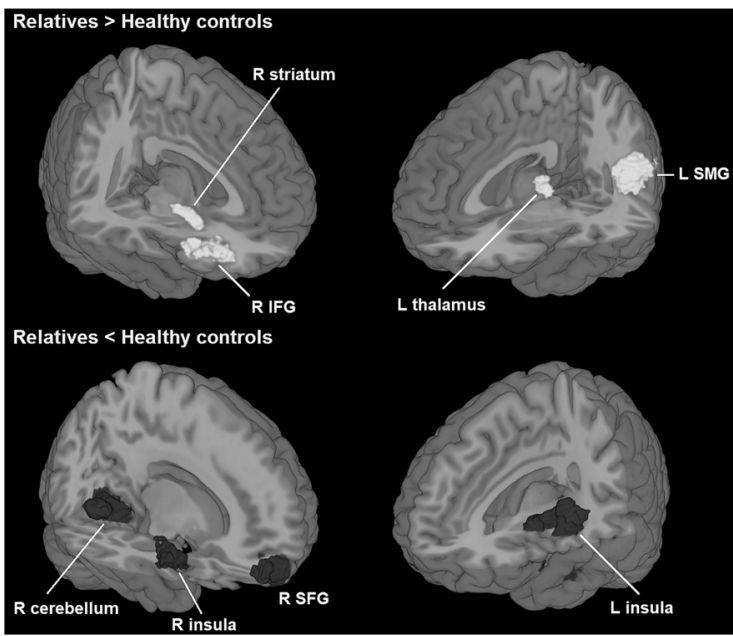

Fig. 2 Meta-analysis of gray matter abnormalities in relatives of patients with a psychiatric disorder. The upper panel with warm color exhibits gray matter increase in relatives, and the lower panel with cold color exhibits gray matter decrease in relatives. IFG inferior frontal gyrus, SFG superior frontal gyrus, SMG supramarginal gyrus, $L$ left, $R$ right.

common and disorder-specific gray matter abnormalities in relatives of patients with $S C Z, B D$, and MDD. Meta-analyses were conducted by combining relatives of patients with all the three disorders in one group, and in subgroup analyses for each separate psychiatric disorder. Following this approach, we identified gray matter reduction in right cerebellum as the only common brain structural abnormality that was present across all sets of analyses. This observation was confirmed with post hoc analyses within the unaffected relative group that removed effects of psychiatric diagnoses in the relatives, and within studies using multiple comparison corrections.

Although previous imaging meta-analyses investigated gray matter abnormalities in relatives of SCZ or BD [21, 22,43,44], our study included a sample larger than before, and also included relatives of patients with MDD, which has not been performed to date. This expanded the effort to identify transdiagnostic features across these three psychiatric disorders. Only one study has investigated common brain structural deficits in relatives of patients with SCZ and BD [22], where cerebellar gray matter deficit was reported as a risk marker for SCZ, but not for BD [22]. As opposed to our study, which used a voxel-wise approach that could identify discrete and localized clusters of gray matter changes, that study compared global measures of cerebellar GMV, providing less information about localized differences within cerebellum subregions.

Assessing both common and disorder-specific brain structural abnormalities in a voxel-based whole brain approach revealed that some gray matter abnormalities might be characteristic of specific disorders. For instance, the increased gray matter in the left thalamus, and decreased gray matter in bilateral insula, seen in relatives of patients with SCZ, were not observed in relatives of patients with BD or MDD in the disorder-specific analyses. Such observations suggest that certain gray matter abnormalities might be a correlation of familial risk for SCZ rather than risk for BD or MDD. Likewise, increased gray matter in left supramarginal gyrus, right inferior frontal gyrus, and decreased gray matter in right superior frontal gyrus might be a correlation of familial risk for BD, and increased gray matter in right striatum a correlation of familial risk to MDD. Of note, we did not compare the relatives of each category against each other; therefore, the assumption of disorder-specific gray matter abnormalities has not been directly tested. In addition, other factors could influence these results, such as power differences in each analysis (there are fewer studies in MDD than in SCZ, for instance). Therefore, these findings need replication by future research.

Common gray matter abnormalities present in relatives

Decreased gray matter in the right cerebellum stands out as the only abnormality across the three groups of relatives included in our meta-analyses. Meta-regression analyses indicated that this abnormality was not significantly affected by sex or other methodological variables. Current views of cerebellar function emphasize its homeostatic role in motor function and affect regulation $[45,46]$. The cerebellum is extensively interconnected with brain regions that are important in cognition and behavior, including prefrontal cortex and limbic cortex [47, 48]. Structural cerebellar deficits, which would cause difficulty in prioritizing, processing, coordinating, and responding to information [49], have also been found in patients with BD [50, 51], SCZ [52, 53], MDD [54], and other psychiatric conditions [55], as well as in our comparisons between patients versus HC. Motor control deficits have been observed across disorders, consistent with the observed structural cerebellar abnormalities [56-58]. Metaanalysis of drug-naive individuals with prodromal psychosis or first-episode psychosis also reported cerebellar gray matter reduction as a significant change in relation to $\mathrm{HC}$ [59]. Moreover, functional deficits in right cerebellum have been identified in meta-analyses in relatives of SCZ [60] and BD patients [61]. Taken together, our finding supports the role of cerebellar abnormalities in the ethiopathogenesis of major psychiatric disorders.

Our findings are also consistent with recent genetic studies pointing to the cerebellum as a brain region of shared vulnerability to mental illness. A recent gene-set and tissue-type gene expression profile analysis reported that an association between shared gene expression profile and brain tissue is the strongest in the cerebellum as compared with other brain regions, supporting the role of the cerebellum in vulnerability to multiple psychiatric disorders [62]. Another study found that the "p factor," a putative behavioral marker of shared liability to psychiatric disorders, is associated with structural changes in the cerebellum, suggesting that the higher the predisposition to psychopathology, the lower the GMV in the cerebellum [63].

Disorder-specific gray matter abnormalities in relatives In relatives of SCZ, increased gray matter in the left thalamus and decreased gray matter in the bilateral insula were identified. The thalamus has a key role in the integration and coordination of information as it passes between various brain regions, disturbance in which could explain the production of psychotic symptoms [64]. Although increased volume has also been implicated in high-risk subjects for SCZ [65], reduced thalamic volume has been mostly found in first-degree relatives [22, 60], and patients with SCZ [66]. Potential compensatory mechanisms in high-risk subjects, which may become exhausted after the illness onset, might explain these discrepancies in the direction of results. This is also supported by our findings that patient probands had decreased gray matter in left thalamus compared with $\mathrm{HC}$ in our secondary analysis. On the contrary, the volumetric reduction in insula has been consistently found in relatives $[60,67]$. It is a region that processed emotional and interoceptive stimuli that underlie self-awareness [68]. In addition, the decreased gray matter in left insula also extended to left middle and superior temporal regions. The middle temporal gyrus is involved in semantic memory processing and language processes $[69,70]$, while superior temporal gyrus is essential for auditory processing [71]. Abnormalities in these temporal regions may account for cognitive deficits in semantic function, and be related to auditory and higher cognitive function alterations in SCZ [72-74]. Previous studies also indicated cortical thinning and 


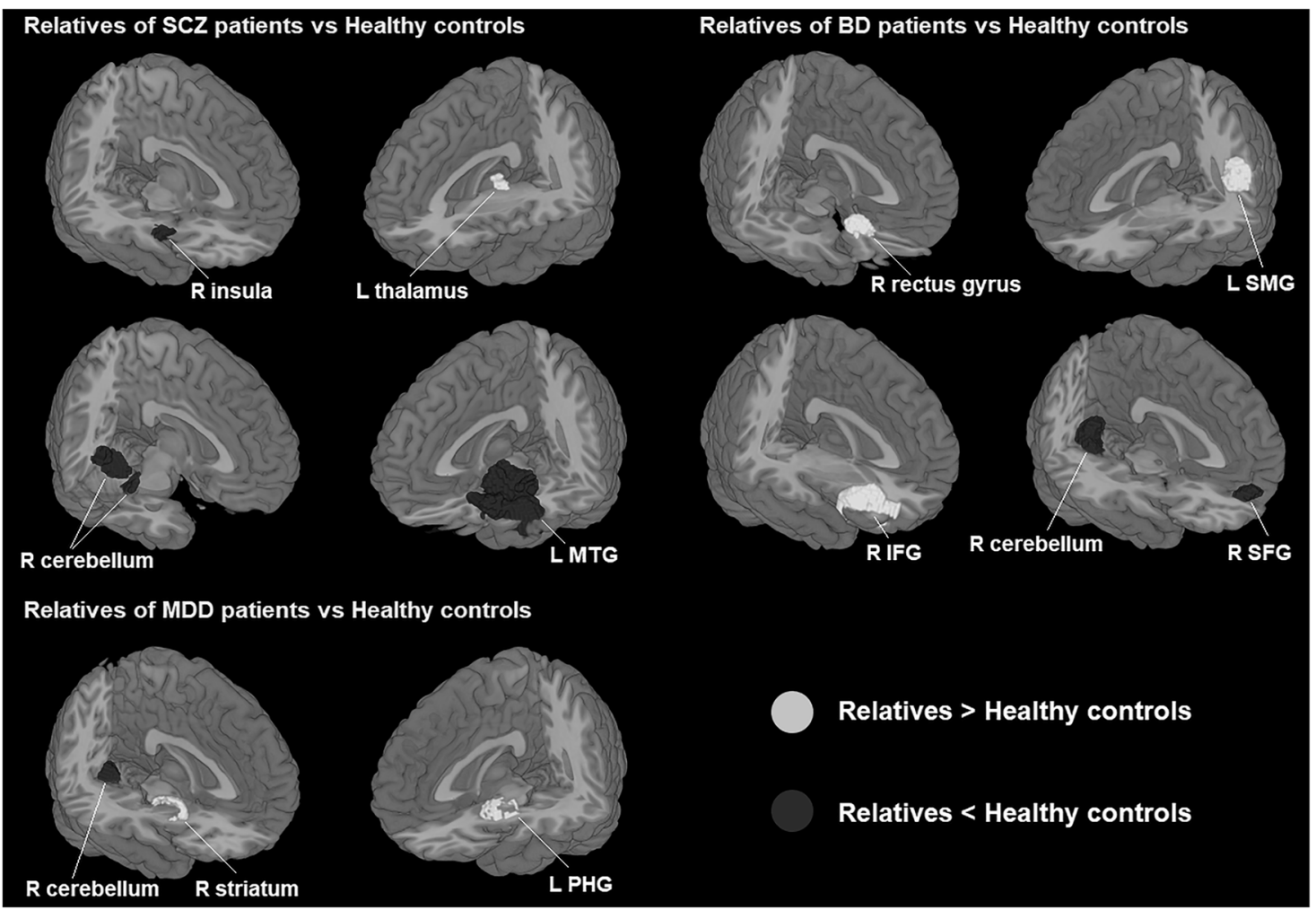

Fig. 3 Subgroup meta-analysis of gray matter abnormalities in relatives of patients with different psychiatric disorders. BD bipolar disorder, MDD major depressive disorder, SCZ schizophrenia, IFG inferior frontal gyrus, MTG middle temporal gyrus, PHG parahippocampal gyrus, SFG superior frontal gyrus, SMG supramarginal gyrus, L left, R right.

gray matter reduction of temporal regions in individuals at high risk for psychosis [75, 76]. Thus, our findings of gray matter decrease in insula and temporal regions may be related to familial risk for SCZ.

Relatives of patients with BD exhibited increased gray matter in left supramarginal gyrus, right inferior frontal gyrus, and decreased gray matter in right superior frontal gyrus, suggesting that these abnormalities might be specific to familial risk for BD. The supramarginal gyrus includes somatosensory association cortex and contains mirror neurons, and plays roles in sensorimotor integration, motor planning and action and basic linguistic functions $[77,78]$. While relatives of patients with BD showed gray matter increase in supramarginal gyrus [39, 79], gray matter deficits in this region were reported in patients with $\mathrm{BD}$ [80]. Thus, increased GMV in the left supramarginal gyrus may represent a resilience marker to disease expression rather than a vulnerability marker in the high-risk individuals for BD. The inferior frontal gyrus is an important area for speech and language [81], and is involved with attention and inhibitory control, specifically inhibition of motor responses, reflexive re-orientation of attention, and updating action plans [82]. Larger right inferior frontal gyrus has also been reported as a biological risk factor for BD in another recent meta-analysis including only relatives of patients with $\mathrm{BD}$ [21]. The superior frontal gyrus is involved in selfawareness and gray matter deficits in superior frontal gyrus have been found in previous meta-analyses of patients with BD [83]. These cortical gray matter abnormalities may represent specific correlates of familial risk for BD.

Gray matter increase in the right striatum was seen in the combined analyses of all the three disorders, but was observed only in the subgroup analysis of relatives of patients with MDD. The striatum plays an important role in cognitive processing and is part of several neuroanatomic circuits associated with emotional processing and regulation [84, 85]. Imaging studies have reported decreased striatal volume in patients with $\operatorname{MDD}[86,87]$. While the potential neurobiological mechanism for the enlargement of striatum in relatives of patients with MDD remains uncertain, our finding highlights a potential critical role of striatum in familial vulnerability of MDD. This finding should be considered with caution due to the small sample size of the included MDD studies. While this is the first meta-analytic report of brain structural abnormalities in relatives of patients with MDD and the finding is promising, further research is needed to confirm this finding and investigate its causes.

Heterogeneity, publication bias, and regression implications We observed a significant, small-to-moderate, between-study heterogeneity effect for the right inferior frontal gyrus, right striatum, left thalamus, and right insula, while no significant between-study heterogeneity was identified in the cerebellar alterations. Between-study heterogeneity in right inferior frontal gyrus showed that $33.1 \%$ of the variance between studies could reflect actual differences in effect sizes in this brain area. Metaregression analysis further showed significant effects of sex, field strength, and slice thickness on the right inferior frontal gyrus, suggesting that differences between studies in these factors might in part explain this heterogeneity. Sex differences in GMV have been found in patients with $\mathrm{SCZ}, \mathrm{BD}$, or MDD [88-91]. A recent meta-analysis in patients with MDD showed that studies with a smaller proportion of males had greater GMV abnormalities in prefrontal regions [83], suggesting that female patients might have greater frontal gray matter abnormalities, which is partly consistent with our finding in family members of patients with psychiatric disorders.

Lower field strength MRI scanner and larger slice thickness during data acquisition were also associated with more gray matter abnormalities in right inferior frontal gyrus. Lower signal noise ratio and spatial resolution can cause partial volume effects and loss of image details. While the precise mechanisms underlying the interaction between clinical and methodological variables and gray 
matter abnormalities in right inferior frontal gyrus remains uncertain, future studies with higher field strength of MRI scanner and smaller slice thickness may clarify alterations in this region.

No evidence of publication bias on any of the identified significant regional clusters was identified by the Egger's test. As always in meta-analyses, studies with negative findings may have not been published, which leads to caution in interpreting our findings.

\section{Limitations}

Several limitations are noteworthy when interpreting our findings. First, we included only voxel-based morphometry studies in this meta-analysis, and our findings were limited to GMV or GMD abnormalities. There is also heterogeneity in methodological details (i.e., different versions of software), and their influences on our findings could not be determined. Second, some relatives were receiving psychotropic medications at time of study which may affect our results, but information about medication treatment was absent or limited in most studies. Hence, medication effects on gray matter cannot be investigated. Third, nearly half of the studies did not assess mood or psychotic symptoms in relatives, and in the other half, symptom rating scales varied widely. Education level and intelligence quotient were also available just for a small portion of included studies. Thus, meta-regression or correlation analyses between gray matter abnormalities and these clinical variables could not be performed. Forth, information about psychotic symptoms or disorders in relatives of patients with mood disorders was also lacking thus we could not investigate their effects. Fifth, some studies might include schizoaffective disorder as SCZ originally, lumping these two disorders together. Therefore, we cannot determine how applicable our findings are for relatives of patients with schizoaffective disorder. Sixth, we did not include the probands in the current study since many efforts have been made in identifying gray matter changes in patients with different psychiatric disorders [17-19]. In addition, one-third of the original studies included in this meta-analysis did not include probands' imaging data $(N=$ 11). Finally, we have not included other psychiatric disorders with high heritability such as attention-deficit/hyperactivity disorder (ADHD) and autism spectrum disorders, which also share genetic liability with SCZ and BD [62]. However, given that recent findings show an association between cerebellar structural deficit and risk for developing autism [92], future research could examine whether decreased gray matter in the right cerebellum is also a correlate of familial risk in those disorders.

In conclusion, our voxel-based whole brain meta-analysis of structural imaging studies suggests that relatives of patients with $\mathrm{SCZ}, \mathrm{BD}$, and MDD exhibit both common and disorder-specific gray matter abnormalities as correlates of familial risk for mental illness. Reduced right cerebellar gray matter might represent a brain structural correlate of a shared genetic vulnerability for mental illness across different psychiatric disorders, while gray matter abnormalities in the neocortex, striatum, and thalamus appear to be disorder-specific. Future research should examine whether right cerebellar structural deficits also characterize familial risk in other highly heritable psychiatric disorders such as autism or ADHD [93].

\section{FUNDING AND DISCLOSURE}

This work was supported by the Fundamental Research Funds for the Central Universities (Grant No. 2020SCU12053), Postdoctoral Interdisciplinary Research Project of Sichuan University (Grant No. 0040204153248), the National Natural Science Foundation of China (Grant Nos. 81671664, 81621003, and 81820108018), and 1.3.5 Project for Disciplines of Excellence, West China Hospital,
Sichuan University (Project No. ZYYC08001 and ZYJC18020). SL also acknowledges the support from Humboldt Foundation Friedrich Wihelm Bessel Research Award.

FGN's spouse is an employee of Eli Lilly \& Co. MPD has received research support from Amarex, Johnson \& Johnson, Pfizer, Otsuka, Shire, Sunovion, Supernus, and Lundbeck. She is a consultant to Akili, CMEology, Johnson \& Johnson, Lundbeck, Neuronetics, Pfizer, Sunovion, Supernus, and Takeda. All other authors report no competing interests.

\section{AUTHOR CONTRIBUTIONS}

FGN, WZ, JAS, and SL contributed to the conception and design of the study. WZ and FGN contributed to paper searching. WZ, SL, JZ, MX, and MJT contributed to data extraction. WZ and LY contributed to the statistical analysis and WZ, JAS, QG, DelBello, SL, and FGN contributed to the interpretation of data. WZ, JAS, SL, and FGN contributed to the drafting of the manuscript, while all authors made critical revision of the manuscript for important intellectual content and gave final approval of the version to be published. WZ, JAS, QG, and SL obtained funding to support this work. WZ, SL, and FGN had full access to all the data of the study and take responsibility for the integrity of the data and the accuracy of the data analysis.

\section{ADDITIONAL INFORMATION}

Supplementary Information accompanies this paper at (https://doi.org/10.1038/ s41386-020-0687-y).

Publisher's note Springer Nature remains neutral with regard to jurisdictional claims in published maps and institutional affiliations.

\section{REFERENCES}

1. Sullivan PF, Kendler KS, Neale MC. Schizophrenia as a complex trait: evidence from a meta-analysis of twin studies. Arch Gen Psychiatry. 2003;60:1187-92.

2. Craddock N, Sklar P. Genetics of bipolar disorder. Lancet. 2013;381:1654-62.

3. Sullivan PF, Neale MC, Kendler KS. Genetic epidemiology of major depression: review and meta-analysis. Am J Psychiatry. 2000;157:1552-62.

4. Bienvenu OJ, Davydow DS, Kendler KS. Psychiatric 'diseases' versus behavioral disorders and degree of genetic influence. Psychol Med. 2011;41:33-40.

5. Kirov G, O'Donovan MC, Owen MJ. Finding schizophrenia genes. J Clin Investig. 2005;115:1440-8.

6. Weissman MM, Gershon ES, Kidd KK, Prusoff BA, Leckman JF, Dibble E, et al. Psychiatric disorders in the relatives of probands with affective disorders. The Yale University-National Institute of Mental Health Collaborative Study. Arch Gen Psychiatry. 1984;41:13-21.

7. Chen MH, Hsu JW, Huang KL, Su TP, Li CT, Lin WC, et al. Risk and coaggregation of major psychiatric disorders among first-degree relatives of patients with bipolar disorder: a nationwide population-based study. Psychol Med. 2019;49:2397-404.

8. Cheng CM, Chang WH, Chen MH, Tsai CF, Su TP, Li CT, et al. Co-aggregation of major psychiatric disorders in individuals with first-degree relatives with schizophrenia: a nationwide population-based study. Mol Psychiatry. 2018;23:1756-63.

9. Rasic D, Hajek T, Alda M, Uher R. Risk of mental illness in offspring of parents with schizophrenia, bipolar disorder, and major depressive disorder: a metaanalysis of family high-risk studies. Schizophr Bull. 2014;40:28-38.

10. Lee SH, Ripke S, Neale BM, Faraone SV, Purcell SM, Perlis RH, et al. Genetic relationship between five psychiatric disorders estimated from genome-wide SNPs. Nat Genet. 2013;45:984-94.

11. Caspi A, Houts RM, Belsky DW, Goldman-Mellor SJ, Harrington H, Israel S, et al. The $p$ factor: one general psychopathology factor in the structure of psychiatric disorders? Clin Psychol Sci. 2014;2:119-37.

12. McGorry $P$, Nelson $B$. Why we need a transdiagnostic staging approach to emerging psychopathology, early diagnosis, and treatment. JAMA Psychiatry. 2016;73:191-2.

13. Krueger RF, Eaton NR. Transdiagnostic factors of mental disorders. World Psychiatry. 2015;14:27-9.

14. Clementz BA, Sweeney JA, Hamm JP, Ivleva El, Ethridge LE, Pearlson GD, et al. Identification of distinct psychosis biotypes using brain-based biomarkers. Am J Psychiatry. 2016;173:373-84.

15. Shah C, Zhang W, Xiao Y, Yao L, Zhao Y, Gao X, et al. Common pattern of graymatter abnormalities in drug-naive and medicated first-episode schizophrenia: a multimodal meta-analysis. Psychol Med. 2017;47:401-13. 
16. Gong Q, Lui S, Sweeney JA. A selective review of cerebral abnormalities in patients with first-episode schizophrenia before and after treatment. Am J Psychiatry. 2016;173:232-43.

17. Goodkind M, Eickhoff SB, Oathes DJ, Jiang Y, Chang A, Jones-Hagata LB, et al. Identification of a common neurobiological substrate for mental illness. JAMA Psychiatry. 2015;72:305-15.

18. Chang M, Womer FY, Edmiston EK, Bai C, Zhou Q, Jiang X, et al. Neurobiological commonalities and distinctions among three major psychiatric diagnostic categories: a structural MRI study. Schizophr Bull. 2018;44:65-74.

19. Gong Q, Scarpazza C, Dai J, He M, Xu X, Shi Y, et al. A transdiagnostic neuroanatomical signature of psychiatric illness. Neuropsychopharmacology. 2019;44:869-75.

20. Nery FG, Monkul ES, Lafer B. Gray matter abnormalities as brain structural vulnerability factors for bipolar disorder: a review of neuroimaging studies of individuals at high genetic risk for bipolar disorder. Aust N Z J Psychiatry. 2013;47:1124-35.

21. Cattarinussi G, Di Giorgio A, Wolf RC, Balestrieri M, Sambataro F. Neural signatures of the risk for bipolar disorder: a meta-analysis of structural and functional neuroimaging studies. Bipolar Disord. 2019;21:215-27.

22. de Zwarte SMC, Brouwer RM, Agartz I, Alda M, Aleman A, Alpert Kl, et al. The association between familial risk and brain abnormalities is disease specific: an ENIGMA-relatives study of schizophrenia and bipolar disorder. Biol Psychiatry. 2019;86:545-56.

23. Fusar-Poli $P$, Smieskova R, Serafini G, Politi P, Borgwardt S. Neuroanatomical markers of genetic liability to psychosis and first episode psychosis: a voxelwise meta-analytical comparison. World J Biol Psychiatry. 2014;15:219-28.

24. Stroup DF, Berlin JA, Morton SC, Olkin I, Williamson GD, Rennie D, et al. Metaanalysis of observational studies in epidemiology: a proposal for reporting. Meta-analysis of Observational Studies in Epidemiology (MOOSE) group. JAMA. 2000;283:2008-12.

25. Nery FG, Norris M, Eliassen JC, Weber WA, Blom TJ, Welge JA, et al. White matter volumes in youth offspring of bipolar parents. J Affect Disord. 2017;209:246-53.

26. Hajek T, Cullis J, Novak T, Kopecek M, Blagdon R, Propper L, et al. Brain structural signature of familial predisposition for bipolar disorder: replicable evidence for involvement of the right inferior frontal gyrus. Biol Psychiatry. 2013;73:144-52.

27. Lui S, Deng W, Huang X, Jiang L, Ouyang L, Borgwardt SJ, et al. Neuroanatomical differences between familial and sporadic schizophrenia and their parents: an optimized voxel-based morphometry study. Psychiatry Res. 2009;171:71-81.

28. Guo W, Hu M, Fan X, Liu F, Wu R, Chen J, et al. Decreased gray matter volume in the left middle temporal gyrus as a candidate biomarker for schizophrenia: a study of drug naive, first-episode schizophrenia patients and unaffected siblings. Schizophr Res. 2014;159:43-50.

29. Lei W, Deng W, Li M, He Z, Han Y, Huang C, et al. Gray matter volume alterations in first-episode drug-naive patients with deficit and nondeficit schizophrenia. Psychiatry Res. 2015;234:219-26.

30. Baiano M, David A, Versace A, Churchill R, Balestrieri M, Brambilla P. Anterior cingulate volumes in schizophrenia: a systematic review and a meta-analysis of MRI studies. Schizophr Res. 2007;93:1-12.

31. Gao X, Zhang W, Yao L, Xiao Y, Liu L, Liu J, et al. Association between structural and functional brain alterations in drug-free patients with schizophrenia: a multimodal meta-analysis. J Psychiatry Neurosci. 2018;43:131-42.

32. Radua J, Borgwardt S, Crescini A, Mataix-Cols D, Meyer-Lindenberg A, McGuire $\mathrm{PK}$, et al. Multimodal meta-analysis of structural and functional brain changes in first episode psychosis and the effects of antipsychotic medication. Neurosci Biobehav Rev. 2012;36:2325-33.

33. Radua J, Schmidt A, Borgwardt S, Heinz A, Schlagenhauf F, McGuire $P$, et al. Ventral striatal activation during reward processing in psychosis: a neurofunctional meta-analysis. JAMA Psychiatry. 2015;72:1243-51.

34. Radua J, Mataix-Cols D, Phillips ML, El-Hage W, Kronhaus DM, Cardoner N, et al. A new meta-analytic method for neuroimaging studies that combines reported peak coordinates and statistical parametric maps. Eur Psychiatry. 2012;27:605-11.

35. Radua J, Rubia K, Canales-Rodriguez EJ, Pomarol-Clotet E, Fusar-Poli $P$, MataixCols D. Anisotropic kernels for coordinate-based meta-analyses of neuroimaging studies. Front Psychiatry. 2014;5:13.

36. Radua J, Mataix-Cols D. Voxel-wise meta-analysis of grey matter changes in obsessive-compulsive disorder. Br J Psychiatry. 2009;195:393-402.

37. Borgwardt SJ, Picchioni MM, Ettinger U, Toulopoulou T, Murray R, McGuire PK. Regional gray matter volume in monozygotic twins concordant and discordant for schizophrenia. Biol Psychiatry. 2010;67:956-64.

38. Matsubara T, Matsuo K, Harada K, Nakano M, Nakashima M, Watanuki T, et al. Distinct and shared endophenotypes of neural substrates in bipolar and major depressive disorders. PLoS ONE. 2016;11:e0168493.

39. Macoveanu J, Baare W, Madsen KH, Kessing LV, Siebner HR, Vinberg M. Risk for affective disorders is associated with greater prefrontal gray matter volumes: a prospective longitudinal study. Neuroimage Clin. 2018;17:786-93.
40. Ivleva El, Clementz BA, Dutcher AM, Arnold SJM, Jeon-Slaughter H, Aslan S, et al. Brain structure biomarkers in the psychosis biotypes: findings from the bipolarschizophrenia network for intermediate phenotypes. Biol Psychiatry. 2017;82:26-39.

41. McIntosh AM, Job DE, Moorhead TW, Harrison LK, Forrester K, Lawrie SM, et al. Voxel-based morphometry of patients with schizophrenia or bipolar disorder and their unaffected relatives. Biol Psychiatry. 2004;56:544-52.

42. Sugranyes G, de la Serna E, Romero S, Sanchez-Gistau V, Calvo A, Moreno D, et al. Gray matter volume decrease distinguishes schizophrenia from bipolar offspring during childhood and adolescence. J Am Acad Child Adolesc Psychiatry. 2015;54:677-84.e2.

43. Xiao Y, Zhang W, Lui S, Yao L, Gong Q. Similar and different gray matter deficits in schizophrenia patients and their unaffected biological relatives. Front Psychiatry. 2013;4:150.

44. Boos HB, Aleman A, Cahn W, Hulshoff Pol H, Kahn RS. Brain volumes in relatives of patients with schizophrenia: a meta-analysis. Arch Gen Psychiatry. 2007;64:297-304

45. Schmahmann JD. The role of the cerebellum in cognition and emotion: personal reflections since 1982 on the dysmetria of thought hypothesis, and its historical evolution from theory to therapy. Neuropsychol Rev. 2010;20:236-60.

46. Schmahmann JD, Caplan D. Cognition, emotion and the cerebellum. Brain. 2006;129:290-2.

47. Schmahmann JD. From movement to thought: anatomic substrates of the cerebellar contribution to cognitive processing. Hum Brain Mapp. 1996;4:174-98.

48. Ramnani N. The primate cortico-cerebellar system: anatomy and function. Nat Rev Neurosci. 2006;7:511-22.

49. Andreasen NC, Paradiso S, O'Leary DS. "Cognitive dysmetria" as an integrative theory of schizophrenia: a dysfunction in cortical-subcortical-cerebellar circuitry? Schizophr Bull. 1998;24:203-18.

50. Eker C, Simsek F, Yilmazer EE, Kitis O, Cinar C, Eker OD, et al. Brain regions associated with risk and resistance for bipolar I disorder: a voxel-based MRI study of patients with bipolar disorder and their healthy siblings. Bipolar Disord. 2014;16:249-61.

51. Kim D, Cho HB, Dager SR, Yurgelun-Todd DA, Yoon S, Lee JH, et al. Posterior cerebellar vermal deficits in bipolar disorder. J Affect Disord. 2013;150:499-506.

52. Wang J, Zhou L, Cui C, Liu Z, Lu J. Gray matter morphological anomalies in the cerebellar vermis in first-episode schizophrenia patients with cognitive deficits. BMC Psychiatry. 2017;17:374.

53. Hirjak D, Wolf RC, Kubera KM, Stieltjes B, Maier-Hein KH, Thomann PA. Neurological soft signs in recent-onset schizophrenia: focus on the cerebellum. Prog Neuropsychopharmacol Biol Psychiatry. 2015;60:18-25.

54. Lai $\mathrm{CH}, \mathrm{Wu}$ YT. The gray matter alterations in major depressive disorder and panic disorder: putative differences in the pathogenesis. J Affect Disord. 2015;186:1-6.

55. Castellanos FX, Lee PP, Sharp W, Jeffries NO, Greenstein DK, Clasen LS, et al. Developmental trajectories of brain volume abnormalities in children and adolescents with attention-deficit/hyperactivity disorder. JAMA. 2002;288:1740-8.

56. Sweeney JA, Luna B, Haas GL, Keshavan MS, Mann JJ, Thase ME. Pursuit tracking impairments in schizophrenia and mood disorders: step-ramp studies with unmedicated patients. Biol Psychiatry. 1999;46:671-80.

57. Lencer R, Sprenger A, Reilly JL, McDowell JE, Rubin LH, Badner JA, et al. Pursuit eye movements as an intermediate phenotype across psychotic disorders: evidence from the B-SNIP study. Schizophr Res. 2015;169:326-33.

58. Sweeney JA, Strojwas MH, Mann JJ, Thase ME. Prefrontal and cerebellar abnormalities in major depression: evidence from oculomotor studies. Biol Psychiatry. 1998:43:584-94.

59. Fusar-Poli $P$, Radua J, McGuire $P$, Borgwardt S. Neuroanatomical maps of psychosis onset: voxel-wise meta-analysis of antipsychotic-naive VBM studies. Schizophr Bull. 2012;38:1297-307.

60. Cooper D, Barker V, Radua J, Fusar-Poli P, Lawrie SM. Multimodal voxel-based meta-analysis of structural and functional magnetic resonance imaging studies in those at elevated genetic risk of developing schizophrenia. Psychiatry Res. 2014;221:69-77.

61. Houenou J, Frommberger J, Carde S, Glasbrenner M, Diener C, Leboyer M, et al. Neuroimaging-based markers of bipolar disorder: evidence from two metaanalyses. J Affect Disord. 2011;132:344-55.

62. Hammerschlag AR, de Leeuw CA, Middeldorp CM, Polderman TJC. Synaptic and brain-expressed gene sets relate to the shared genetic risk across five psychiatric disorders. Psychol Med. 2019;22:1-11.

63. Romer AL, Knodt AR, Houts R, Brigidi BD, Moffitt TE, Caspi A, et al. Structural alterations within cerebellar circuitry are associated with general liability for common mental disorders. Mol Psychiatry. 2018;23:1084-90.

64. Cronenwett WJ, Csernansky J. Thalamic pathology in schizophrenia. Curr Top Behav Neurosci. 2010:4:509-28. 
65. Oertel-Knochel V, Knochel C, Matura S, Rotarska-Jagiela A, Magerkurth J, Prvulovic D, et al. Cortical-basal ganglia imbalance in schizophrenia patients and unaffected first-degree relatives. Schizophr Res. 2012;138:120-7.

66. van Erp TG, Hibar DP, Rasmussen JM, Glahn DC, Pearlson GD, Andreassen OA et al. Subcortical brain volume abnormalities in 2028 individuals with schizophrenia and 2540 healthy controls via the ENIGMA consortium. Mol Psychiatry. 2016;21:547-53.

67. Honea RA, Meyer-Lindenberg A, Hobbs KB, Pezawas L, Mattay VS, Egan MF, et al. Is gray matter volume an intermediate phenotype for schizophrenia? A voxelbased morphometry study of patients with schizophrenia and their healthy siblings. Biol Psychiatry. 2008;63:465-74.

68. Wylie KP, Tregellas JR. The role of the insula in schizophrenia. Schizophr Res. 2010;123:93-104.

69. Martin A, Chao LL. Semantic memory and the brain: structure and processes Curr Opin Neurobiol. 2001;11:194-201.

70. Kiehl KA, Smith AM, Mendrek A, Forster BB, Hare RD, Liddle PF. Temporal lobe abnormalities in semantic processing by criminal psychopaths as revealed by functional magnetic resonance imaging. Psychiatry Res. 2004;130:297-312.

71. Allen P, Laroi F, McGuire PK, Aleman A. The hallucinating brain: a review of structural and functional neuroimaging studies of hallucinations. Neurosci Biobehav Rev. 2008;32:175-91.

72. Kuroki N, Shenton ME, Salisbury DF, Hirayasu Y, Onitsuka T, Ersner-Hershfield H, et al. Middle and inferior temporal gyrus gray matter volume abnormalities in first-episode schizophrenia: an MRI study. Am J Psychiatry. 2006;163:2103-10.

73. Alderson-Day B, Diederen K, Fernyhough C, Ford JM, Horga G, Margulies DS, et al. Auditory hallucinations and the brain's resting-state networks: findings and methodological observations. Schizophr Bull. 2016;42:1110-23.

74. Allen P, Modinos G, Hubl D, Shields G, Cachia A, Jardri R, et al. Neuroimaging auditory hallucinations in schizophrenia: from neuroanatomy to neurochemistry and beyond. Schizophr Bull. 2012;38:695-703.

75. Jung WH, Kim JS, Jang JH, Choi JS, Jung MH, Park JY, et al. Cortical thickness reduction in individuals at ultra-high-risk for psychosis. Schizophr Bull. 2011;37:839-49.

76. Takahashi T, Wood SJ, Yung AR, Soulsby B, McGorry PD, Suzuki M, et al. Progressive gray matter reduction of the superior temporal gyrus during transition to psychosis. Arch Gen Psychiatry. 2009;66:366-76.

77. Reed CL, Caselli RJ. The nature of tactile agnosia: a case study. Neuropsychologia. 1994;32:527-39.

78. Caspers S, Schleicher A, Bacha-Trams M, Palomero-Gallagher N, Amunts K, Zilles $\mathrm{K}$. Organization of the human inferior parietal lobule based on receptor architectonics. Cereb Cortex. 2013;23:615-28.

79. Saricicek A, Yalin N, Hidiroglu C, Cavusoglu B, Tas C, Ceylan D, et al. Neuroanatomical correlates of genetic risk for bipolar disorder: a voxel-based morphometry study in bipolar type I patients and healthy first degree relatives. J Affect Disord. 2015;186:110-8.

80. Li M, Cui L, Deng W, Ma X, Huang C, Jiang L, et al. Voxel-based morphometric analysis on the volume of gray matter in bipolar I disorder. Psychiatry Res. 2011;191:92-7.

81. Zhang W, Li S, Wang X, Gong Y, Yao L, Xiao Y, et al. Abnormal dynamic functional connectivity between speech and auditory areas in schizophrenia patients with auditory hallucinations. Neuroimage Clin. 2018;19:918-24.

82. Levy BJ, Wagner AD. Cognitive control and right ventrolateral prefrontal cortex: reflexive reorienting, motor inhibition, and action updating. Ann N Y Acad Sci. 2011;1224:40-62.

83. Wise T, Radua J, Via E, Cardoner N, Abe O, Adams TM, et al. Common and distinct patterns of grey-matter volume alteration in major depression and bipolar disorder: evidence from voxel-based meta-analysis. Mol Psychiatry. 2017;22:1455-63.

84. Jung $\mathrm{WH}$, Jang JH, Park JW, Kim E, Goo EH, Im OS, et al. Unravelling the intrinsic functional organization of the human striatum: a parcellation and connectivity study based on resting-state FMRI. PLoS ONE. 2014;9:e106768.

85. Lindquist KA, Wager TD, Kober H, Bliss-Moreau E, Barrett LF. The brain basis of emotion: a meta-analytic review. Behav Brain Sci. 2012;35:121-43.

86. Kempton MJ, Salvador Z, Munafo MR, Geddes JR, Simmons A, Frangou S, et al. Structural neuroimaging studies in major depressive disorder. Meta-analysis and comparison with bipolar disorder. Arch Gen Psychiatry. 2011;68:675-90.

87. Lu Y, Liang H, Han D, Mo Y, Li Z, Cheng Y, et al. The volumetric and shape changes of the putamen and thalamus in first episode, untreated major depressive disorder. Neuroimage Clin. 2016;11:658-66.

88. Lang XE, Zhu D, Zhang G, Du X, Jia Q, Yin G, et al. Sex difference in association of symptoms and white matter deficits in first-episode and drug-naive schizophrenia. Transl Psychiatry. 2018;8:281.
89. Ochoa S, Usall J, Cobo J, Labad X, Kulkarni J. Gender differences in schizophrenia and first-episode psychosis: a comprehensive literature review. Schizophr Res Treat. 2012;2012:916198.

90. Yang X, Peng Z, Ma X, Meng Y, Li M, Zhang J, et al. Sex differences in the clinical characteristics and brain gray matter volume alterations in unmedicated patients with major depressive disorder. Sci Rep. 2017;7:2515.

91. Najt $\mathrm{P}$, Nicoletti $\mathrm{M}$, Chen HH, Hatch JP, Caetano SC, Sassi RB, et al. Anatomical measurements of the orbitofrontal cortex in child and adolescent patients with bipolar disorder. Neurosci Lett. 2007;413:183-6.

92. Pote I, Wang S, Sethna V, Blasi A, Daly E, Kuklisova-Murgasova M, et al. Familial risk of autism alters subcortical and cerebellar brain anatomy in infants and predicts the emergence of repetitive behaviors in early childhood. Autism Res. 2019;12:614-27.

93. Mosconi MW, Wang Z, Schmitt LM, Tsai P, Sweeney JA. The role of cerebellar circuitry alterations in the pathophysiology of autism spectrum disorders. Front Neurosci. 2015;9:296.

94. Chen MC, Hamilton JP, Gotlib IH. Decreased hippocampal volume in healthy girls at risk of depression. Arch Gen Psychiatry. 2010;67:270-6.

95. Amico F, Meisenzahl E, Koutsouleris N, Reiser M, Moller HJ, Frodl T. Structural MRI correlates for vulnerability and resilience to major depressive disorder. J Psychiatry Neurosci. 2011;36:15-22.

96. Romanczuk-Seiferth N, Pohland L, Mohnke S, Garbusow M, Erk S, Haddad L, et al. Larger amygdala volume in first-degree relatives of patients with major depression. Neuroimage Clin. 2014;5:62-8.

97. Ozalay O, Aksoy B, Tunay S, Simsek F, Chandhoke S, Kitis O, et al. Cortical thickness and VBM in young women at risk for familial depression and their depressed mothers with positive family history. Psychiatry Res Neuroimaging. 2016;252:1-9.

98. Ladouceur CD, Almeida JR, Birmaher B, Axelson DA, Nau S, Kalas C, et al. Subcortical gray matter volume abnormalities in healthy bipolar offspring: potential neuroanatomical risk marker for bipolar disorder? J Am Acad Child Adolesc Psychiatry. 2008;47:532-9.

99. Kempton MJ, Haldane M, Jogia J, Grasby PM, Collier D, Frangou S. Dissociable brain structural changes associated with predisposition, resilience, and disease expression in bipolar disorder. J Neurosci. 2009;29:10863-8.

100. Lin K, Xu G, Wong NM, Wu H, Li T, Lu W, et al. A multi-dimensional and integrative approach to examining the high-risk and ultra-high-risk stages of bipolar disorder. EBioMedicine. 2015;2:919-28.

101. Nery FG, Gigante AD, Amaral JA, Fernandes FB, Berutti M, Almeida KM, et al. Gray matter volumes in patients with bipolar disorder and their first-degree relatives. Psychiatry Res. 2015;234:188-93.

102. Hanford LC, Hall GB, Minuzzi L, Sassi RB. Gray matter volumes in symptomatic and asymptomatic offspring of parents diagnosed with bipolar disorder. Eur Child Adolesc Psychiatry. 2016;25:959-67.

103. Job DE, Whalley HC, McConnell S, Glabus M, Johnstone EC, Lawrie SM. Voxelbased morphometry of grey matter densities in subjects at high risk of schizophrenia. Schizophr Res. 2003;64:1-13.

104. Tian L, Meng C, Yan H, Zhao Q, Liu Q, Yan J, et al. Convergent evidence from multimodal imaging reveals amygdala abnormalities in schizophrenic patients and their first-degree relatives. PLoS ONE. 2011;6:e28794.

105. Boos HB, Cahn W, van Haren NE, Derks EM, Brouwer RM, Schnack HG, et al. Focal and global brain measurements in siblings of patients with schizophrenia. Schizophr Bull. 2012;38:814-25.

106. Hu M, Li J, Eyler L, Guo X, Wei Q, Tang J, et al. Decreased left middle temporal gyrus volume in antipsychotic drug-naive, first-episode schizophrenia patients and their healthy unaffected siblings. Schizophr Res. 2013;144:37-42.

107. Roman-Urrestarazu A, Murray GK, Barnes A, Miettunen J, Jaaskelainen E, Maki P, et al. Brain structure in different psychosis risk groups in the Northern Finland 1986 birth cohort. Schizophr Res. 2014;153:143-9.

108. Guo W, Song Y, Liu F, Zhang Z, Zhang J, Yu M, et al. Dissociation of functional and anatomical brain abnormalities in unaffected siblings of schizophrenia patients. Clin Neurophysiol. 2015;126:927-32.

109. van der Velde J, Gromann PM, Swart M, de Haan L, Wiersma D, Bruggeman R, et al. Grey matter, an endophenotype for schizophrenia? A voxel-based morphometry study in siblings of patients with schizophrenia. J Psychiatry Neurosci. 2015;40:207-13.

110. Wagshal D, Knowlton BJ, Cohen JR, Bookheimer SY, Bilder RM, Fernandez VG, et al. Cognitive correlates of gray matter abnormalities in adolescent siblings of patients with childhood-onset schizophrenia. Schizophr Res. 2015;161:345-50.

111. Pergola G, Trizio S, Di Carlo P, Taurisano P, Mancini M, Amoroso N, et al. Grey matter volume patterns in thalamic nuclei are associated with familial risk for schizophrenia. Schizophr Res. 2017;180:13-20. 